\title{
Optimal derivatives design for mean-variance agents under adverse selection
}

\author{
Guillaume Carlier * $\quad$ Ivar Ekeland ${ }^{\dagger} \quad$ Nizar Touzi $^{\ddagger}$
}

February 14, 2007

\begin{abstract}
We consider a problem of derivatives design under asymmetry of information: the principal sells a contingent claim to an agent, the type of whom he does not know. More precisely, the principal designs a contingent claim and prices it for each possible agent type, in such a way that each agent picks the contingent claim and pays the price that the principal designed for him. We assume that the preferences of the agent depend linearly on the parameters which determine the agent's type; this model is rich enough to accommodate quadratic utilities. The problem then is reformulated as an optimization problem, where the optimization is performed within a class of convex functions. We prove an existence result for the general case when agents have several characteristics, and we provide explicit examples in the case when the agent is fully characterized by a single parameter
\end{abstract}

Acknowledgements : The authors are grateful for helpful discussions with Elyès Jouini and Walter Schachermayer. The authors thank an anonymous referee whose many suggestions led to substantial improvements of the paper.

Key words : derivatives design, adverse selection, calculus of variations.

\section{Introduction}

Standardized contingent claims, such as futures contracts, bonds, stocks, and derivatives, are traded at market prices in financial markets. There is a well-developed theory dedicated

\footnotetext{
*CEREMADE, Université Paris Dauphine, carlier@ceremade.dauphine.fr.

${ }^{\dagger}$ Canada Research Chair in Mathematical Economics, University of British Columbia, ekeland@math.ubc.ca.

${ }^{\ddagger}$ Centre de Mathématiques Appliquées, Ecole Polytechnique Paris, touzi@cmap.polytechnique.fr, and Imperial College London, n.touzi@ic.ac.uk.
} 
to the pricing and hedging issues of such contingent claims. Standard references are [10] and [4], but there are many more.

This is basically a theory of efficient markets without arbitrage opportunities. It does not address the pricing of so-called structured securities. These are non-standard contingent claims which are tailor-made for some economic agents (individual or firm) facing risk. Major financial institutions routinely sell structured securities to agents who cannot or will not go to the financial markets directly (more about this in the last section of the paper). This puts the agents at an informational disadvantage, which the firm will try to exploit when designing and pricing its product. For instance, the firm, when offering a structured security to an agent, would like to extract the maximum price from the agent, that is, would like to reduce him to his reservation utility. From the point of view of the firm, this would be the first-best situation. However, this may not be possible, either because the agent will hide his characteristics to the firm, or because the agent may prefer another product available to him, and priced so that he gets off with more than his reservation utility. Such a product may be available, either from another firm, or from the same firm, which may have designed and priced it for another customer. In fact, every structured security the firm designs is in competition with the others. In this paper, we will deal with the monopoly situation, where there is a single firm (the principal) the agents can trade with. The principal then has to design its line of products and price them so as to achieve second-best optimum. This is of course a standard situation in economics, but to the best of our knowledge it has not yet been applied to the design of structured securities.

In the following, we formulate a model of optimal derivatives design for a financial institution (the principal) dealing with a set of potential customers (the agents). The principal is in a monopoly situation, and each agent has a reservation utility: agents do not have to buy if their reservation utility is not met, but if they buy, they have to buy from the principal. Each agent is characterized by the values of a set of parameters (initial endowment, risk aversion, and so forth), which he knows, but which may or may not be known to the principal. In both cases, the distribution of types is known to the principal. He then designs a set of structured securities, and posts them with the corresponding prices. Note that the pricing is inherently nonlinear: if the principal offers $X$ at price $p$, he is under no obligation to offer $2 X$ at price $2 p$, nor indeed to offer $2 X$ at all. This imposes some restriction on trading operations: each agent is allowed to trade only once with the firm, and cannot come back for more, so that one cannot buy separately $X$ and $X$ in order to achieve $2 X$; second-hand trading among agents is assumed not to occur. This is more or less reasonable according to the way one interprets the model (see below), but we will not be going into that. The principal then maximizes his profit under the constraint that the agent accepts his offer. This is the so-called adverse selection situation.

There are two interpretations to the above setup. Either the firm is dealing with a single 
customer of unknown type: in this case, the firm has an a priori probability distribution of the type of the agent it is dealing with. Or the firm is dealing with several customers, but cannot deal with them separately, so that all of them are aware of the offers made to the others. In the latter case, it does not matter whether the firm knows the type of each customer or not.

This paper belongs to two distinct lines of research, finance on the one hand, the economics of asymmetric information on the other. On the financial side, there is an extensive literature on securities design. We refer to Duffie and Rahi [11] for a survey article. As we are only interested in the negotiation between two agents, we do not model general equilibrium models. In the case where there is no private information, so that the firm is aware of the true preferences of its potential customer, our problem reduces to the so-called Pareto optimal contracts with the individual rationality constraint. Here again there is a huge literature, see e.g. Bühlmann, and Jewell [5], Gerber [12] and the recent papers in financial mathematics by Barrieu and ElKaroui [2], Jouini, Schachermayer and Touzi [14], and Ruschendorf [24]. On the other hand, none of this literature takes into account the fact that the client may want to hide its true preferences to strengthen his or her negotiating position, or that the firm may have to post its prices to the public, so that any client can take advantage of an offer that was made to another one. Here we have to resort to the economics of asymmetric information, otherwise known as contract theory. We refer to the books of Bolton and Dewatripont [3], Kreps [15], Laffont and Martimort [16], Salanié [25] for an account of the general theory. The standard model in this line of research is the principal-agent model. There are two parties, the principal and the agent, one of whom has private information. The principal moves first, and offers a menu of contracts to the agent. The agent picks one of the contracts on the menu, or declines all of them. If the principal is the informed party, it is a signaling model, as in Akerlof [1] or Spence [26], if the agent is the informed party, then it is a screening model as in Mussa and Rosen [17], and Rochet and Choné [21].

Our model is very much inspired by the one in [21]. The firm moves first, so it is the principal, and it is the uninformed party, so we have a screening model. As usual in the principal-agent framework, this is a monopoly situation: the agent has to buy from the firm, or not at all (in contrast, the paper by Rothschild and Stiglitz [23], describes an equilibrium situation). The private information of the agent concerns his/her type, described by a parameter $\theta$. As in [21], this parameter will be assumed to by multidimensional. It is natural, for instance, to characterize an investor by his/her exposure to risk and his/her risk aversion. Except in particularly simple cases, this requires several parameters. One such case occurs when the agents are assumed to have quadratic utilities: in that case, the risk aversion can be described by a single parameter.

However, the problem we end up with is very different for the one in [21], and indeed in 
any one we have encountered in the contract theory literature. This is due to the fact that, in this literature, and particularly in [21], it is usually assumed that the agent's utility function takes the form:

$$
u(\theta, x)=\theta^{\prime} x+u(x)
$$

that is, it separates into a type-independent part $u(x)$, and a bilinear term $\theta^{\prime} x$. We make much more realistic assumptions on the utility functions. Indeed, $x$ will be replaced by $X$, a random variable, and we will consider as a typical example the mean-variance case:

$$
u(\lambda, X)=\mathbb{E}[X]-\lambda \mathbb{V} \operatorname{ar}[X]
$$

The type $\theta=(\lambda, Y)$ of an agent will be his/her risk aversion coefficient $\lambda$ and his portfolio $Y$, so that his/her utility becomes:

$$
u(\theta, X)=\mathbb{E}[X+Y]-\lambda \mathbb{V} \operatorname{Var}[X+Y]
$$

We shall take a finite-dimensional parametric representation for $Y$, writing $Y=\sum_{i=1}^{k} \beta_{i} B_{i}$, where the $\left(B_{i}\right)_{1 \leq i \leq k}$ 's are prescribed portfolios, so that the type $\theta$ becomes finite-dimensional, $\theta=\left(\lambda, \beta_{1}, \ldots, \beta_{k}\right)$, but even with this reduction the utility function:

$$
u\left(\lambda, \beta_{1}, \ldots, \beta_{k}, X\right)=\mathbb{E}\left[X+\sum_{i=1}^{k} \beta_{i} B_{i}\right]-\lambda \operatorname{Var}\left[X+\sum_{i=1}^{k} \beta_{i} B_{i}\right]
$$

is very far from being of the simple form (1.1). The advantage is clear: the parameters $\lambda$ and $\beta_{i}$ 's can be readily interpreted in economics terms. There is, however, a price to pay: the mathematics of the problem become much harder. Whereas in [21], Rochet and Choné end up with a mathematically simple problem (minimizing a positive definite quadratic functional over a closed convex cone), so that they can prove that the solution exists and is unique without much trouble, in the present paper we end up with minimizing a nonconvex functional, which is not even coercive (i.e. it does not go to infinity at infinity, so it is not clear that minimizing sequences are bounded, let alone that they converge). Nevertheless, we are able to prove that a solution exists (but not that it is unique), thanks to non-standard mathematical techniques. Taking advantage of the simple mathematical form of their problem, Rochet and Choné were able to give an explicit solution in a special, two-dimensional case. Since our problem is much more complicated, we are unable to do so; instead we solve it in one-dimensional situations.

This may be the place for us to discuss the question of dimension. Most papers in adverse selection deal either with finitely many types (typically only two, high and low), or with a one-dimensional continuum of types. This is clearly unrealistic: investors, for instance, are characterized by their risk aversion and their risk exposure, which gives at least two parameters. But the price to pay for more realistic models is mathematical complexity, or even intractability. Models where the types are characterized by the values of two 
continuous parameters or more lead to difficult mathematical problems, where even the existence of a solution comes into question. Roughly speaking, the optimality conditions no longer can be cast as differential equations: they now become partial differential equations. In the particular framework of [21], this partial differential equation was of a known type (elliptic, linear, with constant coefficients), where off-the-shelf theorems are available, but this is far from being the general case. In our framework, for instance, no such results are available, and existence and uniqueness are very much questions to be answered. The fact that we can show existence (but not uniqueness) is the main result of our paper.

The structure of the paper is as follows. The model is described in Section 2, which also provides a characterization of the incentive compatibility condition in terms of convexity. In Section 3, the Principal's maximization program is reformulated as a (non-concave, non-coercive) problem in the calculus of variations: maximize a certain functional within a set of convex functions. From the mathematical point of view, this is a non-standard problem, and Section 4 is devoted to proving that a solution exists. This existence result is interesting in itself, for it does not follow from usual existence arguments, but relies heavily on the fact that optimal contracts are related to convex functions. As stated earlier, we do not know what the solution looks like in the two-dimensional case, so in Section 5, we focus on simpler one-dimensional cases, where either the risk exposure or the risk aversion of the agent is known to the principal, and we give explicit solutions. The last section discusses the significance of our results and describes future directions for research.

\section{Problem formulation}

Let $(\Omega, \mathcal{F}, P)$ be a probability space. Financial assets, including the structured securities which the principal will design, are modeled as contingent claims, that is, random variables on $(\Omega, \mathcal{F}, P)$. They will be assumed to be square-integrable, ie we will restrict our attention to $X \in \mathbb{L}^{2}(\Omega, \mathcal{F}, \mathbb{P})$. In this section, we describe a principal-agent model where the agents have private information on both their risk aversion parameter and their initial endowment of risk. We first consider the case when agents have mean-variance preferences and then we extend our analysis to a larger class of utility functions.

\subsection{Mean-variance utility}

We are given a family of random variables $\left\{1, B_{1}, \ldots, B_{k}\right\}$ in $\mathbb{L}^{2}(\Omega, \mathcal{F}, P)$ for some integer

$k$. For notational conveniency (and actually without loss of generality), we will assume that $B:=\left(B_{1}, \ldots, B_{k}\right)$ satisfies

$$
\mathbb{E} B=0 \text { and } \operatorname{Var} B=I_{k},
$$

where $I_{k}$ is the identity matrix of $\mathbb{R}^{k}$. 
Each agent is characterized by an initial risk endowment $\tilde{\beta}=\left(\beta_{1}, \ldots, \beta_{k}\right)$ and a risk aversion parameter $\lambda$. The corresponding risk endowment is given by the portfolio $\tilde{\beta} \cdot B:=$ $\sum_{i=1}^{k} \tilde{\beta}_{i} B_{i}$. The objective of the agent is to modify his risk profile by trading with the principal.

For given characteristics $(\lambda, \tilde{\beta}) \in \mathbb{R}_{+} \times \mathbb{R}^{k}$, the utility of the agent from a risk transfer $X$ is defined by the mean-variance criterion :

$$
\begin{aligned}
U(\lambda, \tilde{\beta}, X) & =\mathbb{E}[\tilde{\beta} \cdot B+X]-\lambda \mathbb{V} \operatorname{ar}[\tilde{\beta} \cdot B+X] \\
& =\mathbb{E}[X]-\lambda \mathbb{V} \operatorname{ar}[X]-2 \lambda \tilde{\beta} \mathbb{C o v}[X, B]-\lambda|\tilde{\beta}|^{2}
\end{aligned}
$$

We next set $\beta:=2 \lambda \tilde{\beta}$, and we observe that the agent preferences are not affected by the addition of the constant $-\lambda|\tilde{\beta}|^{2}$ to his utility function (note, however, that this will affect his/her reservation utility). This leads to a reduced form of the utility function :

$$
U(\lambda, \tilde{\beta}, X)=\mathbb{E}[X]-\lambda \mathbb{V} \operatorname{ar}[X]-\beta \cdot \operatorname{Cov}[X, B] .
$$

The aim of this paper is to analyze the risk exchange between the mean-variance agent and the principal in a situation of adverse selection, that is, when the mean-variance agents are heterogeneous. The following are some examples of such heterogeneity.

Example 2.1 Heterogeneous initial risk endowment. The risk-aversion parameter $\lambda$ is constant among the agents and known to the principal. The initial holding of risk, defined by $\beta$, is private information, but the principal knows its distribution. The reduced utility function is an affine function of the parameter $\beta$, and is given by

$$
U_{0}(\beta, X):=\beta \cdot G_{0}(X)+g_{0}(X),
$$

where

$$
G_{0}(X):=-\mathbb{C o v}[X, B] \text { and } g_{0}(X):=\mathbb{E}[X]-\lambda \mathbb{V a r}[X]
$$

Example 2.2 Heterogeneous risk aversion. The initial risk endowment, described by the parameter $\beta$, is constant within the agents' population, and known to the principal. The risk aversion parameter $\lambda$ is private information, and the principal knows its distribution. The reduced utility function is an affine function of the parameter $\lambda$, and is given by

$$
U_{0}(\lambda, X):=\lambda G_{0}(X)+g_{0}(X)
$$

where

$$
G_{0}(X):=-\operatorname{Var}[X] \text { and } g_{0}(X):=\mathbb{E}[X]-\beta \operatorname{Cov}[X, B]
$$


Example 2.3 Heterogeneous risk aversion and initial risk endowment. The Principal knows the joint distribution of the parameters $\theta:=(\lambda, \beta)$. The reduced utility function is an affine function of the parameter $\theta$, and is given by

$$
U_{0}(\theta, X):=\theta \cdot G_{0}(X)+g_{0}(X),
$$

where

$$
G_{0}(X):=-(\mathbb{V a r}[X], \mathbb{C o v}[X, B]) \text { and } g_{0}(X):=\mathbb{E}[X]
$$

\subsection{General preferences}

More generally, we are given a set $\mathcal{X}$ of random variables on $(\Omega, \mathcal{F}, P)$ containing 0 . Here, $\mathcal{X}$ is the set of admissible contingent claims that the principal is allowed to choose from. We then define a utility function on $\mathcal{X}$ by :

$$
U(\theta, X):=\theta \cdot G(X)+g(X)
$$

where $\theta$ is drawn from an open convex subset $\Theta$ of $\mathbb{R}^{n}$, the random variable $X$ lies in $\mathcal{X}$, and $G, g$ are given maps from $\mathcal{X}$ into $\mathbb{R}^{n}$ and $\mathbb{R}$, respectively, with

$$
G(0)=0, \quad g(0)=0 .
$$

The latter condition implies that the reservation utility is zero :

$$
U(\theta, 0)=0 \text { for every } \theta \in \Theta .
$$

The common feature of such utilities is that they depend linearly on the parameters $\theta$, although their dependence on the contingent claim $X$ may be quite general. In the sequel, we shall assume that the image set

$$
\Gamma:=G(\mathcal{X}) \text { is a closed subset of } \mathbb{R}^{n} .
$$

Remark 2.1 Condition (2.4) is satisfied in the mean variance examples, with $\mathcal{X}_{0}:=$ $\mathbb{L}^{2}(\Omega, \mathcal{F}, \mathbb{P})$

(i) In the context of a heterogeneous initial risk endowment of Example 2.1, we have $k=n$ and $\Gamma_{0}:=G_{0}\left(\mathcal{X}_{0}\right)=\mathbb{R}^{n}$.

(ii) In the context of a heterogeneous risk aversion of Example 2.2, we have $\Gamma_{0}:=G_{0}\left(\mathcal{X}_{0}\right)=$ $\mathbb{R}_{-}$.

(iii) In the context of a heterogeneous risk aversion and initial risk endowment of Example 2.3, $n=k+1$ and it follows from the Cauchy-Schwarz inequality that

$$
\Gamma_{0}:=G_{0}\left(\mathcal{X}_{0}\right)=\left\{(x, y) \in \mathbb{R} \times \mathbb{R}^{n-1}: x+|y|^{2} \leq 0\right\},
$$

which is a closed subset of $\mathbb{R}^{n}$. 


\subsection{Derivatives design}

The principal designs and prices contingent claims in order to maximize the expected profit from the exchange with the agent. A derivative contract consists of a random variable, representing the risk transfer, together with a scalar premium representing the price of the contract. In view of the uncertainty on the characteristics of the agent, the principal prepares derivatives contracts depending on the distribution of agents characteristics.

Definition 2.1 Suppose $0 \in \mathcal{X}$. Let $U: \Theta \times \mathcal{X} \longrightarrow \mathbb{R}$ be given with $U(\theta, 0)=0$

(i) A derivatives design is a pair $(X, \pi)$ of maps $\theta \longmapsto\left(X_{\theta}, \pi_{\theta}\right)$ from $\Theta$ to $\mathcal{X} \times \mathbb{R}$.

(ii) A derivatives design $(X, \pi)$ satisfies the $U$-individual rationality condition if

$$
U\left(\theta, X_{\theta}\right)-\pi_{\theta} \geq 0 \text { for all } \theta \in \Theta
$$

(iii) A derivatives design $(X, \pi)$ is $U$-incentive-compatible if

$$
U\left(\theta, X_{\theta}\right)-\pi_{\theta} \geq U\left(\theta, X_{\theta^{\prime}}\right)-\pi_{\theta^{\prime}} \text { for all } \theta, \theta^{\prime} \in \Theta^{2} .
$$

We denote by $\operatorname{IR}(U)$ the collection of all derivatives designs which satisfy the $U$-individual rationality condition, and by $\mathrm{IC}(U)$ the collection of all derivatives design which satisfy the $U$-incentive-compatibility condition.

The individual rationality condition states that the agent is allowed not to participate if it gives him/her less than his/her reservation utility. The incentive-compatibility condition states that agents of type $\theta$ will choose the contract $X_{\theta}, \pi_{\theta}$ which the principal designed for them, that is they will buy (or sell) the derivative $X_{\theta}$ and pay (or get) $\pi_{\theta}$.

Remark 2.2 In an economic setting, it would be natural to require that $U$ satisfies the cash-invariance property, that is,

$$
U(\theta, X-\pi)=U(\theta, X)-\pi
$$

for every $\theta \in \Theta, X \in \mathcal{X}$ and $\pi \in \mathbb{R}$. However, the cash-invariance condition is not needed in the subsequent mathematical analysis.

A $U$-incentive-compatible design is a map from $\Theta$ to $\mathcal{X} \times \mathbb{R}$ satisfying certain conditions. The next proposition shows that one can associate with any $U$-incentive-compatible design $(X, \pi)$ a convex function $v$ on $\Theta$, in such a way that $(X, \pi)$ can be recovered from $v$. In other words, the quest for $(X, \pi)$ can be replaced by a quest for $v$. The simplification is considerable: instead of looking for a map from $\Theta$ into an infinite-dimensional space, one is looking for a real-valued function on $\Theta$. It is the key to solving the problem. This result is well-known in the case of utility functions of the form (1.1), see Rochet [19], but 
it seems to be new in the case of utilities in the form (2.1). We also refer to Carlier [6] for general utilities in a finite-dimensional framework.

Recall that a convex function on an open convex subset of $\mathbb{R}^{n}$ (such as $\Theta$ ) is locally Lipschitz, and differentiable almost everywhere.

Proposition 2.1 Let $(X, \pi)$ be a derivatives design.

(i) If $(X, \pi)$ is $U$-incentive-compatible, then the function

$$
v(\theta):=U\left(\theta, X_{\theta}\right)-\pi_{\theta}, \quad \theta \in \Theta
$$

is convex and $G\left(X_{\theta}\right) \in \partial v(\theta)$ for all $\theta \in \Omega$, in particular $\nabla v(\theta) \in \Gamma$ a.e.

(ii) Conversely, let $v$ be a convex function on $\Theta$ with $\nabla v(\theta) \in \Gamma$ a.e. Then there exists an $U$-incentive-compatible design $(X, \pi)$ such that

$$
G\left(X_{\theta}\right) \in \partial v(\theta) \text { and } \pi_{\theta}=U\left(\theta, X_{\theta}\right)-v(\theta), \forall \theta \in \Theta
$$

Proof (i) If $(X, \pi)$ is incentive-compatible then for all $\theta \in \Theta$ :

$$
v(\theta)=\sup _{\theta^{\prime} \in \Theta} \theta \cdot G\left(X_{\theta^{\prime}}\right)+g\left(X_{\theta^{\prime}}\right)-\pi_{\theta^{\prime}}
$$

which proves that $v$ is convex and, for $\left(\theta, \theta^{\prime}\right) \in \Theta^{2}$,

$$
v\left(\theta^{\prime}\right)-v(\theta) \geq \theta^{\prime} \cdot G\left(X_{\theta}\right)+g\left(X_{\theta}\right)-\pi_{\theta}-v(\theta)=\left(\theta^{\prime}-\theta\right) \cdot G\left(X_{\theta}\right)
$$

which precisely means that $G\left(X_{\theta}\right) \in \partial v(\theta)$. In particular if $v$ is differentiable at $\theta$ then $\nabla v(\theta)=G\left(X_{\theta}\right) \in \Gamma$.

(ii) Conversely, let $v$ be a convex function on $\Theta$ with $\nabla v \in \Gamma$ a.e.. For every $\theta \in \Theta$, there exists a sequence $\theta_{k}$ of points of differentiability of $v$, that converges to $\theta$ and such that $\nabla v\left(\theta_{k}\right) \in \Gamma$ for all $k$. Since $v$ is locally Lipschitz, up to some subsequence, we may assume that $\nabla v\left(\theta_{k}\right)$ converges to some $q \in \Gamma$, it is easy to check that $q \in \partial v(\theta)$. We deduce that for every $\theta \in \Theta$ there exists $X_{\theta} \in \mathcal{X}$ such that $G\left(X_{\theta}\right) \in \partial v(\theta)$. Define then $\pi_{\theta}:=\theta \cdot G\left(X_{\theta}\right)+g\left(X_{\theta}\right)-v(\theta)$ for all $\theta \in \Theta$. Since $G\left(X_{\theta^{\prime}}\right) \in \partial v\left(\theta^{\prime}\right)$ for all $\theta^{\prime} \in \Theta$, it follows that $U\left(\theta, X_{\theta}\right)-\pi_{\theta}=v(\theta) \geq v\left(\theta^{\prime}\right)+\left(\theta-\theta^{\prime}\right) \cdot G\left(X_{\theta^{\prime}}\right)=U\left(\theta, X_{\theta^{\prime}}\right)-\pi_{\theta^{\prime}}$, hence $(X, \pi)$ is $U$-incentive-compatible.

Suppose that a derivatives design $(X, \pi)$ is $U$-incentive-compatible. It follows from the definition of $v$ that $(X, \pi)$ is also $U$-individually rational if and only if:

$$
v(\theta) \geq 0, \quad \forall \theta \in \Theta
$$

This observation will be useful in the next section. 


\subsection{The optimal design problem}

Let $C(X)$ be the cost incurred to the principal for issuing the contingent claim $X$. So $C$ is a real-valued function on $\mathcal{X}$, the set of admissible securities. We assume that $C(0)=0$.

The profit realized by the principal from trading with agent $\theta$ is then given by

$$
\pi_{\theta}-C\left(X_{\theta}\right)
$$

As noted in Remark 2.2, it would be natural to assume that $C$ is cash-invariant, but this is not needed for further developments.

We next assume that the characteristics $\theta$ of the agent are distributed according to an absolutely continuous (with respect to Lebesgue) measure on $\Theta$, with density $\mu$. The principal seeks to design a derivatives contract $(X, \pi)$ that will maximize his expected profit, subject to the incentive-compatibility and the rational individuality conditions. The precise formulation is then as follows :

$$
\Phi:=\sup _{(X, \pi) \in \mathcal{D}(U)} \int_{\Theta}\left[\pi_{\theta}-C\left(X_{\theta}\right)\right] \mu(\theta) d \theta
$$

where $\mathcal{D}(U)$ is the collection of all derivatives designs in $\operatorname{IR}(U) \cap \operatorname{IC}(U)$ such that $(\pi-C \circ X)$ is $\mu$-measurable and the negative part of $(\pi-C \circ X)$ belongs to $\mathbb{L}^{1}(\mu)$. Let us remark that $\Phi \geq 0$ but need not be finite.

By contrast, the first-best problem, where the principal knows the characteristics of the agents, and no agent has access to another's contract, is given by:

$$
\sup _{(X, \pi) \in \operatorname{IR}(U)} \int_{\Theta}\left[\pi_{\theta}-C\left(X_{\theta}\right)\right] \mu(\theta) d \theta .
$$

\section{Reformulation}

By proposition 2.1, a derivatives design satisfying the individual rationality and incentivecompatibility conditions is characterized by the choice of a convex function in the set

$$
\begin{aligned}
\mathcal{A} & :=\{v: \Theta \longrightarrow \mathbb{R}, v \text { is convex, } \partial v(\theta) \cap \Gamma \neq \emptyset \text { and } v(\theta) \geq 0 \text { for every } \theta \in \Theta\} \\
& =\{v: \Theta \longrightarrow \mathbb{R}, v \text { is convex, } \nabla v \in \Gamma \text { a.e. and } v \geq 0\} .
\end{aligned}
$$

A function $v \in \mathcal{A}$ can be interpreted as the indirect utility of the agent from exchanging with the principal, and corresponds to the set of derivatives designs that satisfy:

$$
\pi_{\theta}=U\left(\theta, X_{\theta}\right)-v(\theta)=\theta \cdot G\left(X_{\theta}\right)+g\left(X_{\theta}\right)-v(\theta), \quad G\left(X_{\theta}\right) \in \partial v(\theta), \theta \in \Theta
$$


If $(X, \pi) \in \mathcal{D}(U)$ and $v \in \mathcal{A}$ satisfy (3.2) then it follows from the absolute continuity of $\mu$ with respect to the Lebesgue measure on $\Theta$ that

$$
\int_{\Theta}\left[\pi_{\theta}-C\left(X_{\theta}\right)\right] \mu(\theta) d \theta=\int_{\Theta}\left[\theta \cdot \nabla v(\theta)-v(\theta)+g\left(X_{\theta}\right)-C\left(X_{\theta}\right)\right] \mu(\theta) d \theta .
$$

In order to reformulate the problem (2.5) in terms of $v$ only, we introduce the function :

$$
H(p):=\inf \{C(X)-g(X): X \in \mathcal{X} \text { and } G(X)=p\} \text { for all } p \in \Gamma \text {. }
$$

Note that $H(0) \leq C(0)-g(0)=0$ by the normalization (2.2). Let us consider the following problem in the calculus of variations:

$$
\sup _{v \in \mathcal{A}} \phi(v), \quad \phi(v):=\int_{\Theta} \varphi(\theta, v(\theta), \nabla v(\theta)) \mu(\theta) d \theta,
$$

where:

$$
\varphi(\theta, r, p):=\theta \cdot p-r-H(p) \text { for }(\theta, r, p) \in \Theta \times \mathbb{R}_{+} \times \Gamma .
$$

Under mild assumptions on $H$, the following statement gives the precise connection between the principal's problem (2.5) and its reformulation (3.4).

Proposition 3.1 Assume that $H$ is lower semi continuous on $\Gamma$ and that for every $p \in \Gamma$, there exists $X \in \mathcal{X}$ such that $G(X)=p$ and $C(X)-g(X)=H(p)$, then:

(i) $\Phi:=\sup _{v \in \mathcal{A}} \phi(v)$,

(ii) if $v \in \mathcal{A}$ satisfies $\Phi=\phi(v)$, then every derivatives design $(X, \pi)$ such that

$$
\begin{array}{r}
G\left(X_{\theta}\right) \in \partial v(\theta), \pi_{\theta}=\theta \cdot G\left(X_{\theta}\right)+g\left(X_{\theta}\right)-v(\theta), \forall \theta \in \Theta, \\
\text { and } C\left(X_{\theta}\right)-g\left(X_{\theta}\right)=H(\nabla v(\theta)) \text { for } \mu \text {-a.e. } \theta \in \theta,
\end{array}
$$

solves the principal's problem (2.5).

(iii) if $(X, \pi)$ solves (2.5), then $v$ defined by:

$$
v(\theta):=\theta \cdot G\left(X_{\theta}\right)+g\left(X_{\theta}\right)-\pi_{\theta}, \forall \theta \in \Theta
$$

solves the calculus of variations problem (3.4).

Proof (i) Let $(X, \pi) \in \mathcal{D}(U)$ and define $v$ by $v(\theta)=\theta \cdot G\left(X_{\theta}\right)+g\left(X_{\theta}\right)-\pi_{\theta}$ for all $\theta \in \Theta$, then $v \in \mathcal{A}$ and $G\left(X_{\theta}\right)=\nabla v(\theta) \mu$-a.e.. We then have:

$$
\pi_{\theta}-C\left(X_{\theta}\right)=\theta \cdot \nabla v(\theta)-v(\theta)+g\left(X_{\theta}\right)-C\left(X_{\theta}\right) \leq \varphi(\theta, v(\theta), \nabla v(\theta)) .
$$

Hence $\Phi \leq \sup _{v \in \mathcal{A}} \phi(v)$. By Proposition 4.1 proved below, for every $\alpha<\sup _{v \in \mathcal{A}} \phi(v)$, there exists $v \in \mathcal{A}$ such that

$$
\phi(v) \geq \alpha \text { and } \varphi(\theta, v(\theta), \nabla v(\theta)) \geq-H(0) \mu \text {-a.e.. }
$$


Let $(X, \pi)$ satisfy $(3.6)$, then $\varphi(\theta, v(\theta), \nabla v(\theta))=\pi_{\theta}-C\left(X_{\theta}\right)$ a.e., $(X, \pi) \in \mathcal{D}(U)$ and

$$
\alpha \leq \phi(v)=\int_{\Theta}\left[\pi_{\theta}-C\left(X_{\theta}\right)\right] \mu(\theta) d \theta \leq \Phi .
$$

since $\alpha<\sup _{v \in \mathcal{A}} \phi(v)$ is arbitrary, we finally have $\Phi=\sup _{v \in \mathcal{A}} \phi(v)$.

(ii) By the a priori estimate of Proposition 4.1, we may further assume that $\varphi(\theta, v(\theta), \nabla v(\theta))$ $\geq-H(0)$ a.e.. If $(X, \pi)$ satisfies $(3.6)$, then $\varphi(\theta, v(\theta), \nabla v(\theta))=\pi_{\theta}-C\left(X_{\theta}\right)$ a.e., $(X, \pi) \in$ $\mathcal{D}(U)$, which yields

$$
\int_{\Theta}\left[\pi_{\theta}-C\left(X_{\theta}\right)\right] \mu(\theta) d \theta=\phi(v)=\Phi
$$

(iii) The statement immediately follows from:

$$
\begin{aligned}
\Phi & =\int_{\Theta}\left[\pi_{\theta}-C\left(X_{\theta}\right)\right] \mu(\theta) d \theta \\
& =\int_{\Theta}\left[\theta \cdot \nabla v(\theta)-v(\theta)+g\left(X_{\theta}\right)-C\left(X_{\theta}\right)\right] \mu(\theta) d \theta \\
& \leq \int_{\Theta}[\theta \cdot \nabla v(\theta)-v(\theta)-H(\nabla v(\theta)] \mu(\theta) d \theta=\phi(v) .
\end{aligned}
$$

By the previous Proposition, the existence and the characterization of an optimal derivatives design (i.e. a solution of (2.5)) is reduced to the variational problem (3.4).

We conclude this section by providing the expression of the function $H$ in the context of a mean variance utility function for the agents. As for the principal, we shall assume that he has access to a complete market. Then the cost function is given by the (unique) arbitrage price of the contingent claim

$$
C_{0}(X)=\mathbb{E}[Z X] \text { for some } Z \in \mathcal{X}_{0} \text {, with } Z>0 \text { and } \mathbb{E}[Z]=1 .
$$

The random variable $Z$ is the density with respect to $P$ of the unique risk-neutral measure of the complete market.

Example 3.1 Heterogeneous initial risk endowment, continued. In this context, the function $H_{0}:=H$ is defined by

$$
H_{0}(p):=\inf \{\mathbb{E}[Z X]-\mathbb{E}[X]+\lambda \mathbb{V a r}[X]: \mathbb{C o v}[X, B]=-p\}
$$

By direct computation, we obtain

$$
H_{0}(p)=\lambda|p|^{2}-p \cdot \mathbb{C o v}[Z, B]-\frac{1}{4 \lambda}\left(\mathbb{V a r}[Z]-\mathbb{C o v}[Z, B]^{2}\right) .
$$


The minimizer in the expression of $H_{0}$ is unique up to a constant (this is a consequence of the cash invariance of the problem), with zero-mean minimizer given by

$$
X_{0}(p)=\frac{1}{2 \lambda}(1-Z+(\mathbb{C o v}[Z, B]-2 \lambda p) \cdot B) .
$$

Setting:

$$
\zeta:=\operatorname{Var}[Z] \text { and } \xi:=\mathbb{C o v}[Z, B]
$$

the principal's problem then becomes:

$$
\begin{aligned}
& \sup \int\left[(\beta+\xi) \cdot \nabla v(\beta)-v(\beta)-\lambda|\nabla v(\beta)|^{2}+\frac{1}{4 \lambda}\left(\zeta-\xi^{2}\right)\right] \mu(\beta) d \beta \\
& v \text { convex, } v \geq 0 .
\end{aligned}
$$

Example 3.2 Heterogeneous risk aversion, continued. In this context, the function $H_{0}:=$ $H$ is defined by

$$
H_{0}(p):=\inf \{\mathbb{E}[Z X]-\mathbb{E}[X]+\beta \cdot \mathbb{C o v}[X, B]: \operatorname{Var}[X]=-p\}
$$

A direct computation yields

$$
H_{0}(p)=-\sqrt{-p \mathbb{V} \operatorname{ar}[Z+\beta \cdot B]},
$$

the minimizer in the expression of $H_{0}$ is unique up to a constant, as a consequence of the cash invariance of the problem, the zero-mean minimizer given by

$$
X_{0}(p)=-\sqrt{\frac{-p}{\operatorname{Var}[Z+\beta \cdot B]}}(Z-1+\beta \cdot B) .
$$

Using the notations (3.8), the principal's problem then becomes:

$$
\begin{aligned}
& \sup \int\left[\lambda v^{\prime}(\lambda)-v(\lambda)+\sqrt{-\left(\zeta+\left|\beta^{2}\right|+2 \beta \cdot \xi\right) v^{\prime}(\lambda)}\right] \mu(\lambda) d \lambda \\
& v \text { convex, } v \geq 0, v^{\prime} \leq 0
\end{aligned}
$$

Example 3.3 Heterogeneous risk aversion and initial risk endowment, continued. In this context, the function $H_{0}:=H$ is defined by

$$
H_{0}(p):=\inf \{\mathbb{E} Z X-\mathbb{E} X:(\mathbb{V a r} X, \mathbb{C o v}[X, B])=-(x, y)\}
$$


where $p=(x, y)$, and we directly compute that

$$
H_{0}(p)=-y \cdot \operatorname{Cov}[Z, B]-\sqrt{-x-|y|^{2}} \sqrt{\mathbb{V a r} Z-\mathbb{C o v}[Z, B]^{2}} \text { for } p=(x, y) \in \Gamma_{0},
$$

and the corresponding zero-mean minimizer is given by

$$
X_{0}(p)=-\sqrt{\frac{-x-|y|^{2}}{\operatorname{Var} Z-\mathbb{C o v}[Z, B]^{2}}}(Z-1-\mathbb{C o v}[Z, B] \cdot B)-y \cdot B .
$$

The principal's problem then becomes:

$$
\begin{aligned}
& \sup \int\left[\lambda \frac{\partial v}{\partial \lambda}+(\beta+\xi) \cdot \frac{\partial v}{\partial \beta}-v(\lambda, \beta)+\sqrt{-\frac{\partial v}{\partial \lambda}-\left|\frac{\partial v}{\partial \beta}\right|^{2}} \sqrt{\zeta-\xi^{2}}\right] \mu(\lambda, \beta) d \lambda d \beta \\
& v \text { convex, } v \geq 0,\left|\frac{\partial v}{\partial \beta}\right|^{2}+\frac{\partial v}{\partial \lambda} \leq 0 .
\end{aligned}
$$

Observe that the integrand is not a concave function of the derivatives $\frac{\partial v}{\partial \beta}$ and $\frac{\partial v}{\partial \lambda}$, and does not go to infinity when the derivatives go to infinity. One may contrast this, for instance, with the situation in [21], where the integrand is a simple quadratic function of the derivatives. For this reason, it is a mathematical challenge to prove that the principal's problem does have a solution. We address this problem in the next section.

\section{Existence result}

\subsection{An a priori estimate}

We shall prove the existence of solutions to (3.4), under mild assumptions. The key point is given by proposition 4.1 which states that if $H(0)=0$, given any derivatives contract such that the principal loses money on certain types of agents, there is another contract which increases the overall profit, and such that the principal now breaks even on all types $\theta \in \Theta$. In other words, if the null contract has zero cost $(H(0)=0)$, cross subsidies among agents are not efficient. Let us explain the economic intuition behind this result (we also refer to [6] for a similar result with nonlinear utilities). Imagine that a derivatives menu makes negative profit for some types and positive for others, then we can firstly remove the bad contracts from the initial menu and secondly offer the bad types their prefered contract in the set of good contracts completed by the null contract. If we do so, we obtain a new incentive-compatible menu that leaves the good agents with their initial contract and forces the initially bad ones to chose a contract which is either the null contract or makes money. Up to some mathematical subtleties, the proof of proposition 4.1 heavily builds upon the previous intuition. From the mathematical point, the assumption $H(0)=0$ plays 
no particular role in the proof (one may change $H$ into $H-H(0)$, provided $H(0)$ is finite, without loss of generality) but is only practical for the previous economic interpretation.

Proposition 4.1 Assume that $H$ is lower semi-continuous on $\Gamma$ and $H(0)=0$. Then for all $v \in \mathcal{A}$ there exists $w \in \mathcal{A}$ such that:

$$
\phi(w) \geq \phi(v) \text { and } \varphi(\theta, w(\theta), \nabla w(\theta)) \geq 0 \text { for a.e. } \theta \in \Theta
$$

where the previous quantities possibly take the value $+\infty$.

Proof For a subset $S \subset \Theta$, we denote by

$$
S^{+}(v):=\{\theta \in S: \nabla v(\theta) \text { exists and } \varphi(\theta, v(\theta), \nabla v(\theta)) \geq 0\} .
$$

If $\Theta^{+}(v)$ has zero Lebesgue measure, i.e. $\lambda\left(\Theta^{+}(v)\right)=0$, then $w=0$ satisfies $\phi(0)=0 \geq$ $\phi(v), \varphi(\theta, 0,0)=0$, and the proof is complete. We then concentrate on the case

$$
\lambda\left(\Theta^{+}(v)\right)>0
$$

1. We first assume in addition that $\Theta$ is bounded and $v$ is Lipschitz-continuous on $\Theta$, and prove that the statement of the proposition holds with

$$
w(\theta):=\max \{0, \tilde{w}(\theta)\}, \quad \tilde{w}(\theta):=\sup _{\theta^{\prime} \in \Theta^{+}(v)}\left\{v\left(\theta^{\prime}\right)+\nabla v\left(\theta^{\prime}\right) \cdot\left(\theta-\theta^{\prime}\right)\right\}, \quad \theta \in \Theta .
$$

Clearly, the function $w$ is convex, $w \leq v$ on $\Theta$, and $w=v$ on $\Theta^{+}(v)$. By the convexity of $v$, and its differentiability on $\Theta^{+}(v)$, this implies that $\partial w(\theta) \subset \partial v(\theta)=\{\nabla v(\theta)\}$ for every $\theta \in \Theta^{+}(v)$. Thus, $w$ is differentiable at each point of $\Theta^{+}(v)$ and $\nabla w=\nabla v$ on $\Theta^{+}(v)$. Then

$$
\nabla w \in \Gamma \text { and } \varphi(\theta, w(\theta), \nabla w(\theta)) \geq 0 \text { for every } \theta \in \Theta^{+}(v)
$$

since these properties are already satisfied by $v$. We shall next prove that

$$
\nabla w \in \Gamma \text { and } \varphi(\theta, w(\theta), \nabla w(\theta)) \geq 0 \text { for a.e. } \theta \in \Theta \backslash \Theta^{+}(v) \text {. }
$$

In view of (4.3), the first claim in (4.4) shows that $w \in \mathcal{A}$, and the second claim implies in particular that $\varphi(\theta, w(\theta), \nabla w(\theta)) \geq \varphi(\theta, v(\theta), \nabla v(\theta))$ for a.e. $\theta \in \Theta$, since $\varphi(\theta, v(\theta), \nabla v(\theta))<0$ a.e. on $\Theta \backslash \Theta^{+}(v)$. This implies that $\phi(w) \geq \phi(v)$, which proves the required claim.

We now turn to the proof of (4.4). Let $\theta \in \Theta \backslash \Theta^{+}(v)$ be a point of differentiability of $w$.

1.1. If $w(\theta)=0, \theta$ is an interior point of minimum for $w$, recall that $\Theta$ is an open subset of $\mathbb{R}^{n}$. Then $\nabla w(\theta)=0$, and (4.4) follows from the fact that $0 \in \Gamma$ and $H(0)=0$. 
1.2. If $w(\theta)>0$, there exists a sequence $\left(\theta_{k}\right) \subset \Theta^{+}(v)$ such that

$$
w(\theta)=\lim _{k \rightarrow \infty} v\left(\theta_{k}\right)+\nabla v\left(\theta_{k}\right) \cdot\left(\theta-\theta_{k}\right) .
$$

Since $\bar{\Theta}$ is compact and $v$ is Lipschitz-continuous on $\Theta$, the sequences $\theta_{k}$ and $\nabla v\left(\theta_{k}\right)$ are bounded. Then, after possibly passing to a subsequence, and using the closure of $\Gamma$, we have

$$
\left(\theta_{k}, \nabla v\left(\theta_{k}\right)\right) \longrightarrow(\bar{\theta}, \bar{p}) \in \bar{\Theta} \times \Gamma
$$

Since $\theta_{k} \in \Theta^{+}(v)$, it follows from the definition of $w$ and (4.5) that

$$
\begin{aligned}
w\left(\theta^{\prime}\right) & \geq \lim _{k \rightarrow \infty} v\left(\theta_{k}\right)+\nabla v\left(\theta_{k}\right) \cdot\left(\theta^{\prime}-\theta_{k}\right) \\
& =w(\theta)+\bar{p}\left(\theta^{\prime}-\theta\right) \text { for every } \theta^{\prime} \in \Theta,
\end{aligned}
$$

Hence $\bar{p} \in \partial w(\theta)=\{\nabla w(\theta)\}$. Since $\bar{p} \in \Gamma$ this shows the first part of (4.4). To see that the second part of (4.4) is also satisfied, let us recall that, since $\theta_{k} \in \Theta^{+}(v)$, one has:

$$
\varphi\left(\theta_{k}, v\left(\theta_{k}\right), \nabla v\left(\theta_{k}\right)\right)=\theta_{k} \cdot \nabla v\left(\theta_{k}\right)-v\left(\theta_{k}\right)-H\left(\nabla v\left(\theta_{k}\right)\right) \geq 0 .
$$

The lower semi-continuity of $H$ and (4.5) then implies

$$
\begin{aligned}
H(\nabla w(\theta)) \leq \liminf _{k \rightarrow \infty} H\left(\nabla v\left(\theta_{k}\right)\right) & \leq \liminf _{k \rightarrow \infty} \theta_{k} \cdot \nabla v\left(\theta_{k}\right)-v\left(\theta_{k}\right) \\
& =-\lim _{k \rightarrow \infty} v\left(\theta_{k}\right)+\nabla v\left(\theta_{k}\right) \cdot\left(\theta-\theta_{k}\right)+\lim _{k \rightarrow \infty} \nabla v\left(\theta_{k}\right) \cdot \theta \\
& =-w(\theta)+\nabla w(\theta) \cdot \theta
\end{aligned}
$$

and the proof of (4.4) is complete.

2. We now drop the compactness condition on the set $\bar{\Theta}$. Let $\left(\Theta_{n}\right)_{n \geq 1}$ be an increasing sequence of compact subsets with $\cup_{n \geq 1} \Theta_{n}=\Theta$. By (4.2), we may assume in addition that $\lambda\left(\Theta_{n}^{+}(v)\right)>0$. For every $n \geq 1$, we set

$$
\begin{aligned}
w_{n}(\theta):=\max \left\{0, \tilde{w}_{n}(\theta)\right\}, \quad \tilde{w}_{n}(\theta):=\sup _{\theta^{\prime} \in \Theta_{n}^{+}(v)}\left\{v\left(\theta^{\prime}\right)+\nabla v\left(\theta^{\prime}\right) \cdot\left(\theta-\theta^{\prime}\right)\right\}, & \theta \in \Theta \\
w(\theta) & :=\max \{0, \tilde{w}(\theta)\}, \quad \tilde{w}(\theta):=\sup _{\theta^{\prime} \in \Theta^{+}(v)}\left\{v\left(\theta^{\prime}\right)+\nabla v\left(\theta^{\prime}\right) \cdot\left(\theta-\theta^{\prime}\right)\right\}, \quad \theta \in \Theta .
\end{aligned}
$$

Since $w_{n}$ is convex for every $n$, we have by construction

$$
\left(w_{n}(\theta), \nabla w_{n}(\theta)\right) \longrightarrow(w(\theta), \nabla w(\theta)) \text { for a.e. } \theta \in \Theta
$$

For each $n \geq 1$, it follows from the previous step that $w_{n} \in \mathcal{A}, \varphi\left(\theta, w_{n}(\theta), \nabla w_{n}(\theta)\right) \geq 0$ and $\varphi\left(\theta, w_{n}(\theta), \nabla w_{n}(\theta)\right) \geq \varphi(\theta, v(\theta), \nabla v(\theta))$ a.e. on $\Theta_{n}$. By the lower semi-continuity of $H$, we then obtain by an immediate passage to the limit that

$$
w \in \mathcal{A}, \quad \varphi(\theta, w(\theta), \nabla w(\theta)) \geq 0 \quad \text { and } \quad \varphi(\theta, w(\theta), \nabla w(\theta)) \geq \varphi(\theta, v(\theta), \nabla v(\theta)) \geq 0
$$

a.e. on $\Theta$, and the proof is complete. 


\subsection{Existence of optimal derivatives design}

In order to prove the main result of this paper, let us extend $H$ on $\mathbb{R}^{n}$ by setting $H=+\infty$ outside $\Gamma$, and introduce its Legendre-Fenchel transform

$$
H^{*}(\theta):=\sup _{p \in \mathbb{R}^{n}}(p \cdot \theta-H(p))=\sup _{p \in \Gamma}(p \cdot \theta-H(p)),
$$

Since $H(0) \leq 0$, it follows that $H^{*} \geq 0$. Throughout, we adopt the convention $\sup \emptyset=$ $-\infty$.

Theorem 4.1 Let $H$ be a lower semi-continuous function on $\Gamma$, and assume that $H^{*} \in$ $\mathbb{L}^{1}(\mu)$ and

$$
\limsup _{|p| \rightarrow \infty}(p \cdot \theta-H(p))<-H(0) \quad \text { for every } \theta \in \Theta .
$$

Then $\Phi<\infty$ and existence holds for the principal problem defined in (3.4), i.e. there exists $v \in \mathcal{A}$ such that

$$
\phi(v) \geq \phi(w), \forall w \in \mathcal{A} \text {. }
$$

Proof 1. By possibly adding a constant to $H$, we may assume that $H(0)=0$. Since $H$ is assumed to be lower semi-continuous, we deduce from proposition 4.1 that there exists a sequence $\left(v_{n}\right)_{n \geq 1} \subset \mathcal{A}$ such that

$$
\Phi:=\sup _{v \in \mathcal{A}} \phi(v)=\lim _{n \rightarrow \infty} \phi\left(v_{n}\right)
$$

and

$$
\theta \cdot \nabla v_{n}(\theta)-H\left(\nabla v_{n}(\theta)\right) \geq v_{n}(\theta) \geq 0 \text { for all } n \geq 1 \text { and a.e. } \theta \in \Theta \text {. }
$$

2. For $r \geq 0$, let $B_{r}$ denote the ball of center 0 and radius $r$, and define the function

$$
\gamma(\theta, r):=\sup _{p \in \Gamma \backslash B_{r}}(p \cdot \theta-H(p))
$$

Clearly, $\gamma$ is convex in $\theta$, non-increasing in $r, \gamma(\theta, 0)=H^{*}(\theta) \geq 0$ and by assumption (4.6), for every $\theta \in \Theta$, there exists $r=r(\theta)>0$ such that $\gamma(\theta, r)<0$. Then the function

$$
\rho(\theta):=\inf \{r>0: \gamma(\theta, r)<0\}, \quad \theta \in \Theta,
$$

is finite. From the convexity of $\gamma$ in $\theta$, it can readily be checked that $\rho$ is quasi-convex, i.e. $\rho\left(\lambda \theta_{1}+\left(1-\lambda \theta_{2}\right) \leq \max \left\{\rho\left(\theta_{1}\right), \rho\left(\theta_{2}\right)\right\}\right.$.

3. It follows from (4.8) that

$$
\left|\nabla v_{n}(\theta)\right| \leq \rho(\theta) \text { and } 0 \leq v_{n}(\theta) \leq \theta \cdot \nabla v_{n}(\theta)-H\left(\nabla v_{n}(\theta)\right) \leq H^{*}(\theta), \theta \in \Theta \text { a.e. }
$$


Since $\rho$ is finite and quasi-convex on $\Theta$, it is in fact locally bounded on $\Theta$, this implies that $v_{n}$ and $\nabla v_{n}$ are locally uniformly bounded in $\Theta$. By Ascoli's Theorem, we deduce that (up to some subsequence) the sequence $\left(v_{n}\right)_{n \geq 1}$ converges uniformly on compact subsets of $\Theta$ to some (convex nonnegative) function $v$, after possibly passing to a subsequence. By convexity, we also have the a.e. convergence of $\nabla v_{n}$ towards $\nabla v$, which implies that the limit function $v \in \mathcal{A}$.

4. We also deduce from (4.8) that

$$
\begin{aligned}
0 \leq \varphi\left(\theta, v_{n}(\theta), \nabla v_{n}(\theta)\right) & =\quad \theta \cdot \nabla v_{n}(\theta)-v_{n}(\theta)-H\left(\nabla v_{n}(\theta)\right) \\
\leq & \theta \cdot \nabla v_{n}(\theta)-H\left(\nabla v_{n}(\theta)\right) \leq H^{*}(\theta) .
\end{aligned}
$$

This shows that

$$
\Phi=\lim _{n \rightarrow \infty} \phi\left(v_{n}\right) \leq \int_{\Theta} H^{*}(\theta) \mu(\theta) d \theta<\infty .
$$

It follows from Fatou's Lemma that

$$
\begin{aligned}
\Phi & =\lim _{n \rightarrow \infty} \int_{\Theta} \varphi\left(\theta, v_{n}(\theta), \nabla v_{n}(\theta)\right) \mu(\theta) d \theta \\
& \leq \int_{\Theta} \limsup _{n \rightarrow \infty} \varphi\left(\theta, v_{n}(\theta), \nabla v_{n}(\theta)\right) \mu(\theta) d \theta \leq \phi(v),
\end{aligned}
$$

which shows that $\Phi=\phi(v)$.

\subsection{Application to mean-variance agents}

We now show that the existence result of Theorem 4.1 applies to our examples of meanvariance agents when the Principal has access to a complete market. We shall denote

$$
\zeta:=\operatorname{Var}[Z] \text { and } \xi:=\operatorname{Cov}[Z, B]
$$

Example 4.1 Heterogeneous initial risk endowment, continued. We recall that in this case $\lambda>0$ is fixed and:

$$
H_{0}(p)=\lambda|p|^{2}-p \cdot \xi-\frac{1}{4 \lambda}\left(\zeta-|\xi|^{2}\right) .
$$

It therefore follows from Theorem 4.1 that there exists an optimal derivatives design provided $\int_{\mathbb{R}^{n}}|\beta|^{2} \mu(\beta) d \beta<\infty$. Observe that, in this case, Problem (3.4) satisfies an usual coercivity in the calculus of variations and by strict convexity, its solution is unique. Let us also remark that, in this example, problem (3.4) has the same mathematical structure as the problem studied by Rochet and Choné in [21]. 
Example 4.2 heterogeneous risk aversion, continued. $\quad$ In this case $\beta \in \mathbb{R}^{n}$ is fixed and:

$$
H_{0}(p)=-\sqrt{-p \mathbb{V a r}[Z+\beta B]} .
$$

Setting $\chi:=\operatorname{Var}(Z+\beta \cdot B)$, we have

$$
\lim _{p \rightarrow-\infty} p \lambda-H_{0}(p)=\lim _{p \rightarrow-\infty} p \lambda+\sqrt{-\chi p}=-\infty \text { for every } \lambda>0 .
$$

Moreover, the Legendre-Fenchel transform is given by

$$
H_{0}^{*}(\lambda):=\frac{\chi}{4 \lambda} \text { for } \lambda>0
$$

We then conclude by Theorem 4.1 that existence holds if the characteristics distribution $\mu$ satisfies $\int_{0}^{\infty}(\mu(\lambda) / \lambda) d \lambda<\infty$.

Example 4.3 heterogeneous risk aversion and initial risk endowment, continued. In this case, we denote $\theta=(\lambda, \beta) \in \Theta_{0} \subset \mathbb{R}_{+} \times \mathbb{R}^{n-1}, p=(x, y) \in \Gamma_{0} \subset \mathbb{R}_{-} \times \mathbb{R}^{n-1}$ and recall that:

$$
H_{0}(x, y)=-y \cdot \xi-\alpha \sqrt{-x-|y|^{2}} \text { with } \alpha:=\sqrt{\zeta-|\xi|^{2}} \geq 0
$$

Let $\theta=(\lambda, \beta) \in \Theta_{0}$ and let $p=(x, y) \in \Gamma_{0}$ be such that

$$
\lambda x+\beta \cdot y-H_{0}(x, y) \geq 0 .
$$

Using $|y| \leq \sqrt{-x}$ together with the Cauchy-Schwarz inequality, (4.10) yields

$$
\lambda x+(|\beta+\xi|+\alpha) \sqrt{-x} \geq 0 .
$$

Since $x \leq 0$ we get $x \in\left[-\lambda^{-2}(|\beta+\xi|+\alpha)^{2}, 0\right]$. Condition (4.6) of Theorem 4.1 is therefore satisfied.

We next directly compute that

$$
H^{*}(\lambda, \beta)=\sup _{x+|y|^{2} \leq 0} x \lambda+y \cdot \beta+y \cdot \xi+\alpha \sqrt{-x-|y|^{2}}=\frac{\alpha^{2}+|\beta+\xi|^{2}}{4 \lambda},
$$

and we conclude by Theorem 4.1 that existence holds provided that

$$
\int_{\Theta_{0}} \frac{\alpha^{2}+|\beta+\xi|^{2}}{\lambda} \mu(\lambda, \beta) d \lambda d \beta<\infty
$$




\section{Examples of explicit solutions in dimension 1}

\subsection{Heterogeneous initial risk endowment}

We first consider the case when all agents have the same risk aversion $\lambda>0$, but hold different amounts $\widetilde{\beta} \geq 0$ of a single asset $B$ (the market portfolio). Without loss of generality, we assume that $\operatorname{Var}[B]=1$, and, as above, we set $\beta:=2 \lambda \widetilde{\beta}$ to be the type of the agent.

The only characteristic of the agent is his/her initial portfolio $\beta B / 2 \lambda$. We assume that $\beta \in[\underline{\beta}, \bar{\beta}]$, with $\underline{\beta} \geq 0$. The density of types is $\mu(\beta)>0$, and the cumulative distribution function is $P(\beta)$ :

$$
P(\beta):=\int_{\underline{\beta}}^{\beta} \mu(s) d s, \text { with } P(\bar{\beta})=1 .
$$

If agent of type $\beta$ adds $X$ to his portfolio and pays $\pi$, his resulting utility is:

$$
\mathbb{E}\left[\frac{\beta}{2 \lambda} B+X\right]-\lambda \operatorname{Var}\left[\frac{\beta}{2 \lambda} B+X\right]-\pi \text {. }
$$

If the agent does not trade, so that $X=0$ and $\pi=0$, he then gets his reservation utility, namely $\left(2 \beta \mathbb{E}[B]-\beta^{2}\right) / 4 \lambda$. So the individual rationality constraint of type $\beta$ becomes:

$$
\begin{aligned}
& \mathbb{E}\left[\frac{\beta}{2 \lambda} B+X\right]-\lambda \mathbb{V a r}\left[\frac{\beta}{2 \lambda} B+X\right]-\pi \geq \frac{1}{4 \lambda}\left(2 \beta \mathbb{E}[B]-\beta^{2}\right) \\
& \Leftrightarrow \mathbb{E}[X]-\lambda \operatorname{Var}[X]-\beta \operatorname{Cov}(B, X)-\pi \geq 0
\end{aligned}
$$

which is consistent with our earlier notations, by taking the reduced utility function to be:

$$
u(\beta, X):=\mathbb{E}[X]-\lambda \mathbb{V a r}[X]-\beta \mathbb{C O v}[B, X]
$$

and setting the reservation utility to 0 . As above, we assume that the principal has access to a complete market, so that her cost for producing $X$ is $\mathbb{E}[X Z]$ for some positive $Z \in L^{2}$ with $\mathbb{E}[Z]=1$.

Note that both the agent's utility and the principal's cost are cash-invariant: a security $X$ is worth the same as $(X-\mathbb{E}[X])$ plus a cash payment of $\mathbb{E}[X]$, both for the principal and the agent. So the gains from trading $X$ are exactly the same as the gains from trading $(X-\mathbb{E}[X])$. This means that, without loss of generality, we can assume that the agents are trading zero-mean securities:

$$
\mathbb{E}[X]=0
$$

In other words, the agents are trading risk, not cash. Henceforth, we set:

$$
Z_{0}=Z-\mathbb{E}[Z], \quad B_{0}=B-\mathbb{E}[B] .
$$




\subsubsection{The first-best.}

If the principal knows the type $\beta \geq 0$ of the agent, she will offer him his reservation utility. So the first-best problem is:

$$
\sup \{-\lambda \mathbb{V} a r[X]-\beta \mathbb{C o v}[B, X]-\mathbb{E}[X Z]: \mathbb{E}[X]=0\} \text {. }
$$

The solution is:

$$
X(\beta)=-\frac{1}{2 \lambda} Z_{0}-\frac{\beta}{2 \lambda} B_{0}
$$

At the first best, the principal trades with all agents. The security she offers decomposes into two parts: full insurance, namely $-\beta B_{0} / 2 \lambda$, and a fixed part, $-Z_{0} / 2 \lambda$, independent of the type. In other words, the principal pays the agents to produce $-Z_{0} / 2 \lambda$ and resells it on the complete market. After the transaction, all agents bear the same risk, namely $-Z_{0} / 2 \lambda$.

\subsubsection{The second-best.}

As stated in Example 3.1, the reduced version of the second-best problem is given by

$$
\begin{aligned}
& \sup _{\underline{\beta}} \int_{\underline{\beta}}^{\bar{\beta}}\left[\beta v^{\prime}-v-\lambda v^{\prime 2}+\mathbb{C o v}[B, Z] v^{\prime}+\frac{1}{4 \lambda}\left(\mathbb{V a r}[Z]-\mathbb{C o v}[B, Z]^{2}\right)\right] \mu(\beta) d \beta \\
& v \geq 0, v \text { convex. }
\end{aligned}
$$

The constant in the integral plays no role in the optimization, and may be discarded. We end up with:

$$
\begin{aligned}
& \sup _{\int_{\underline{\beta}}^{\bar{\beta}}}^{\bar{\beta}}\left[\beta v^{\prime}-v-\lambda v^{\prime 2}+\mathbb{C o v}[B, Z] v^{\prime}\right] \mu(\beta) d \beta \\
& v \geq 0, v \text { convex. }
\end{aligned}
$$

Changing $v(\beta)$ to $v(\beta)-c$, we increase the value of the integral, until we hit the positivity constraint. From this it follows that $v$ must vanish somewhere on $[\beta, \bar{\beta}]$. Let $x$ be such a value - say for instance it is the greatest - so that $v(x)=0$.

For the sake of simplicity, we assume from now on that the cost of producing $B_{0}$ is non-negative and that types are uniformly distributed:

$$
\operatorname{Cov}[B, Z] \geq 0, \quad \mu(\beta)=\frac{1}{\bar{\beta}-\underline{\beta}}, \quad P(\beta)=\frac{\beta-\underline{\beta}}{\bar{\beta}-\underline{\beta}}
$$

Integrating by parts, the integral to be maximized reduces to: 


$$
\begin{aligned}
& -v(\bar{\beta})+\frac{1}{\bar{\beta}-\underline{\beta}} \int_{\underline{\beta}}^{\bar{\beta}}\left[(2 \beta+\operatorname{Cov}[B, Z]-\underline{\beta}) v^{\prime}-\lambda v^{\prime 2}\right] d \beta= \\
& \frac{1}{\bar{\beta}-\underline{\beta}} \int_{\underline{\beta}}^{x}\left[(2 \beta+\operatorname{Cov}[B, Z]-\underline{\beta}) v^{\prime}-\lambda v^{\prime 2}\right] d \beta+ \\
& \frac{1}{\bar{\beta}-\underline{\beta}} \int_{x}^{\bar{\beta}}\left[(2 \beta+\operatorname{Cov}[B, Z]-\bar{\beta}) v^{\prime}-\lambda v^{\prime 2}\right] d \beta
\end{aligned}
$$

which is to be maximized among all points $x \in[\beta, \bar{\beta}]$ and all increasing functions $v^{\prime}(\beta)$.

The pointwise maximum is achieved for:

$$
v_{1}^{\prime}(\beta)=\frac{1}{2 \lambda}(2 \beta+\operatorname{Cov}[B, Z]-\underline{\beta}) \text { and } v_{2}^{\prime}(\beta)=\frac{1}{2 \lambda}(2 \beta+\operatorname{Cov}[B, Z]-\bar{\beta})
$$

inside the first and second integral respectively. Note that $v_{1}^{\prime}(\beta) \geq 0$ on $[\underline{\beta}, \bar{\beta}]$, while $v_{2}^{\prime}(\underline{\beta}) \leq 0$ if $2 \underline{\beta}+\operatorname{Cov}[B, Z]-\bar{\beta} \leq 0$. This leads us to two distinct cases:

\subsubsection{Case 1: $\operatorname{Cov}[B, Z]>\bar{\beta}-2 \underline{\beta}$}

In that case, the optimal choice is $x=\underline{\beta}$. We have:

$$
v^{\prime}(\beta)=v_{2}^{\prime}(\beta)=\frac{1}{2 \lambda}(2 \beta+\mathbb{C o v}[B, Z]-\bar{\beta})
$$

and hence:

$$
v(\beta)=\frac{1}{2 \lambda}\left(\beta^{2}+(\mathbb{C o v}[B, Z]-\bar{\beta}) \beta+c\right)
$$

The constant $c$ is adjusted by the fact that $v(\underline{\beta})=0$, which yields:

$$
v(\beta)=\frac{1}{2 \lambda}(\beta+\operatorname{Cov}[B, Z]+\underline{\beta}-\bar{\beta})(\beta-\underline{\beta}) .
$$

Note that in that case, $v^{\prime}(\underline{\beta})>0$.

\subsubsection{Case 2: $\operatorname{Cov}[B, Z] \leq \bar{\beta}-2 \underline{\beta}$}

In that case, the optimal choice is given by $v_{2}^{\prime}(x)=0$, so that:

$$
x=\frac{1}{2}(\bar{\beta}-\operatorname{Cov}[B, Z]) .
$$

The maximum in the first integral is achieved for $v_{1}^{\prime}(\beta) \geq 0$, but this leads to $v^{\prime}=v_{1}^{\prime}$ being increasing on $[\underline{\beta}, x]$, and since $v^{\prime}(x)=0$ we would have $v^{\prime}(\beta)<0$, in violation of the positivity constraint. So we must have $v^{\prime}(\beta)=0$ on $[\beta, x]$. The solution is: 


$$
\begin{aligned}
& v^{\prime}(\beta)=0 \text { if } \beta \leq x \\
& v^{\prime}(\beta)=\frac{1}{2 \lambda}(2 \beta+\operatorname{Cov}[B, Z]-\bar{\beta}) \quad \text { if } \beta \geq x
\end{aligned}
$$

which gives $v(\beta)$ up to constant, which is adjusted by setting $v(x)=0$

$$
\begin{aligned}
& v(\beta)=0 \text { if } \beta \leq x \\
& v(\beta)=\frac{1}{2 \lambda}(\beta-x)^{2} \quad \text { if } \beta \geq x .
\end{aligned}
$$

Note that in that case, $v^{\prime}(x)=0$.

\subsubsection{The optimal design}

As noted earlier, we have:

$$
-\operatorname{Cov}[B, X(\beta)]=v^{\prime}(\beta)
$$

recalling Example 3.1, we then have

$$
\begin{aligned}
X(\beta) & =\frac{1}{2 \lambda}\left[-Z_{0}+\left(\mathbb{C o v}[B, Z]-2 \lambda v^{\prime}(\beta)\right) B_{0}\right] \\
& =-\frac{1}{2 \lambda} Z_{0}-\left[v^{\prime}(\beta)-\frac{1}{2 \lambda} \mathbb{C o v}[B, Z]\right] B_{0} \\
\pi(\beta) & =-\lambda \mathbb{V a r}[X(\beta)]-\beta \operatorname{Cov}[B, X(\beta)]-v(\beta) \\
& =\beta v^{\prime}(\beta)-v(\beta)-\frac{1}{4 \lambda} \operatorname{Var}\left[-Z_{0}+\left(\mathbb{C o v}[B, Z]-2 \lambda v^{\prime}(\beta)\right) B_{0}\right]
\end{aligned}
$$

so that:

$$
\begin{aligned}
& X(\beta)=-\frac{1}{2 \lambda} Z_{0}-\frac{1}{2 \lambda}(2 \beta-\bar{\beta}) B_{0} \text { if } \beta \geq \frac{1}{2}(\bar{\beta}-\mathbb{C o v}[B, Z]) \\
& X(\beta)=-\frac{1}{2 \lambda} Z_{0}+\frac{1}{2 \lambda} \operatorname{Cov}[B, Z] B_{0} \text { if } \beta \leq \frac{1}{2}(\bar{\beta}-\mathbb{C o v}[B, Z])
\end{aligned}
$$

In particular, we have:

$$
X(\bar{\beta})=-\frac{1}{2 \lambda} Z_{0}-\frac{1}{2 \lambda} \bar{\beta} B_{0}
$$

and:

$$
X(\underline{\beta})=\left\{\begin{array}{c}
-\frac{1}{2 \lambda} Z_{0}-\frac{1}{2 \lambda}(2 \underline{\beta}-\bar{\beta}) B_{0} \text { if } \underline{\beta} \geq \frac{1}{2}(\bar{\beta}-\mathbb{C o v}[B, Z]) \\
-\frac{1}{2 \lambda} Z_{0}-\frac{1}{2 \lambda} \operatorname{Cov}[B, Z] B_{0} \text { if } \underline{\beta} \leq \frac{1}{2}(\bar{\beta}+\operatorname{Cov}[B, Z])
\end{array}\right.
$$

As usual, there is no distortion at the top, but there is distortion at the bottom: agent $\bar{\beta}$ gets the first-best security, and pays less than the first-best price. On the other hand, the agent with the lowest risk does not get the first-best security. 


\subsection{Heterogeneous risk aversion}

We now consider the case of heterogeneous risk aversion. All agents have the same initial risk endowment equal to zero, but they have heterogenous risk aversion coefficient $\lambda$. The distribution of $\lambda$ is known, and denoted by $\mu$. In this case the optimal design problem amounts to solving the problem:

$$
\sup \left\{I(v):=\int\left[\lambda v^{\prime}-v-H\left(v^{\prime}\right)\right] d \mu: v \geq 0, \text { convex, non-increasing }\right\}
$$

Assuming that the principal has access to a complete market, so that $C(X)=\mathbb{E}[Z X]$, where $Z \in L^{2}, Z \geq 0$ and $\mathbb{E}[Z]=1$, and defining $\zeta:=\mathbb{V a r}[Z]$, we have:

$$
H(p)=\inf \{\mathbb{E}[Z X]-\mathbb{E}[X]: \operatorname{Var}[X]=-p\}=-\sqrt{-p \zeta} .
$$

To fix ideas, we assume that $\mu$ is supported on some interval $[a, b]$ and absolutely continuous with respect to the Lebesgue measure on that interval. We denote by $F$ the cumulative distribution function of $\mu$, so that $F^{\prime}$ is the corresponding probability density function. Let us remark that if $v$ is admissible for (5.1) then $I(v-v(b))=I(v)+v(b) \geq I(v)$ hence the optimal solution must satisfy $v(b)=0$. Defining $w:=v^{\prime}$, it follows from a direct integration by parts that:

$$
I(v)=J(w):=\int_{a}^{b}\left[(\lambda \mu+F) w+\sqrt{-w \zeta} \cdot F^{\prime}\right] d \lambda .
$$

The problem (5.1) therefore simply amounts to maximizing $J$ in the set of nondecreasing, non-positive functions $w$. We then have a concave optimization problem subject to a monotonicity constraint, such problems are standard in adverse selection problems (see Guesnerie-Laffont [13], Rochet [20] or Mussa and Rosen [17]). To solve this problem, let us first define $w_{0}$ as the function that maximizes the integrand in the definition of $J$, forgetting about the negativity and the monotonicity constraints, an elementary computation yields:

$$
w_{0}(\lambda)=-\frac{\zeta}{4}\left(\frac{F^{\prime}(\lambda)}{\lambda F^{\prime}(\lambda)+F(\lambda)}\right)^{2} .
$$

We continue the analysis of this example by considering two cases.

1st case: Observe that $w_{0}$ is nondecreasing as soon as $\lambda \longmapsto \lambda+F(\lambda) / F^{\prime}(\lambda)$ is. The previous condition is satisfied for instance when $\mu$ is uniform or more generally whenever the hazard rate function $F^{\prime} / F$ is non-increasing. In this case, $w_{0}$ characterizes the optimal contract (recall that $-w_{0}(\lambda)$ is the variance of the risk transfer $X_{\lambda}$ chosen by agents with $\lambda$-risk aversion).

2nd case: We next consider the case where $w_{0}$ is not monotone nondecreasing. This leads to the so-called bunching effect i.e. there are intervals of characteristics $\beta$ which are

offered the same contract. The economic interpretation of the bunching phenomenon is 
the following: when the incentive problem is serious, it is too costly for the Principal to offer a discriminating contract. As an example of this phenomenon, let us consider the case where $\zeta=1, a=0, b=1$ and the density is (up to a normalizing constant) given by

$$
F^{\prime}(\lambda)=1-\lambda^{2}\left(1-\lambda^{4}\right)
$$

Then $w_{0}$ is no longer nondecreasing (see figure below) hence cannot be the solution. In other words, the incentive-compatibility condition is binding at the optimum. However, the shape of $w_{0}$ and the so-called ironing procedure (see Rochet [20] or Mussa and Rosen [17] for details) imply that the solution is actually of the form $w^{*}=\min \left(w_{0}, w_{0}\left(\lambda^{*}\right)\right)$ for some $\lambda^{*} \in\left(0, \lambda_{0}\right)$ where $\lambda_{0}$ denotes the maximum point of $w_{0}$. Optimizing with respect to $\lambda^{*}$ yields the optimality condition:

$$
\int_{\lambda^{*}}^{1}\left(\lambda F^{\prime}+F\right) d \lambda=\frac{1}{2 \sqrt{-w_{0}\left(\lambda^{*}\right)}} \int_{\lambda^{*}}^{1} \mu d \lambda
$$

An approximate value $\lambda^{*} \approx 0.815$ is found numerically.

The graphs of $w_{0}$ and of the solution are given in the following figure.

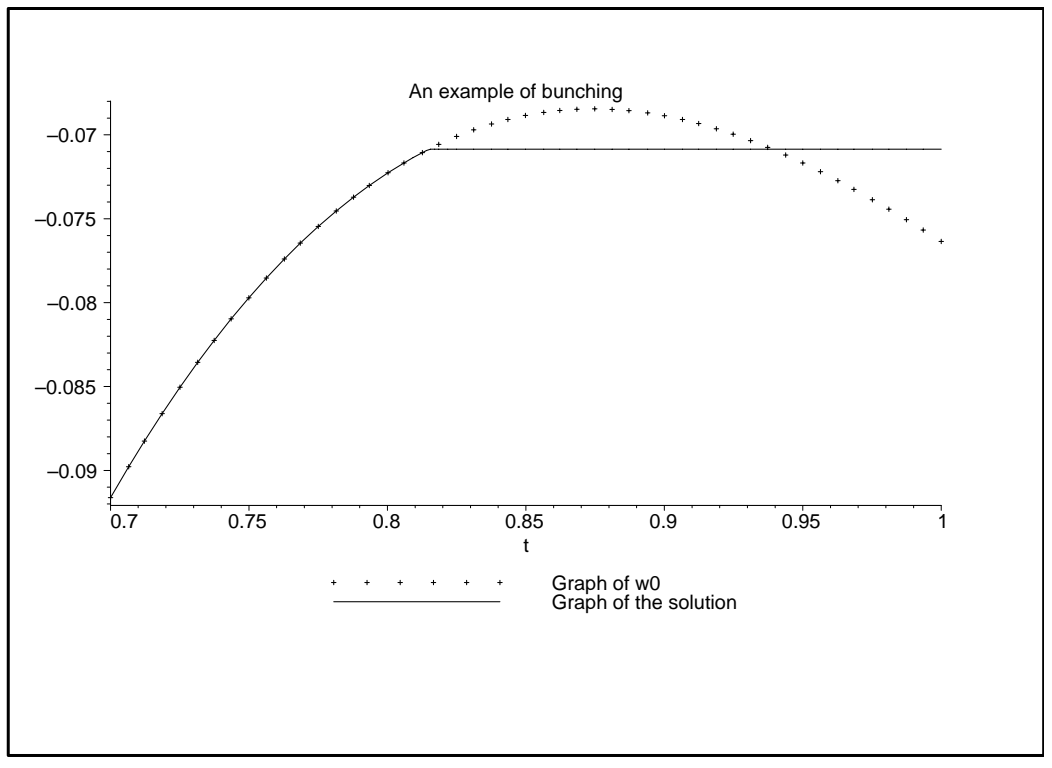

\section{Conclusion}

In conclusion, we would like to raise a number of issues which this paper leaves unsolved.

The first one is mathematical. Although we have stated the principal's problem for utility functions of the type $u(\theta, X)=\theta \cdot G(X)+g(X)$, and we have given an existence result for this general class, we have no qualitative results, and no efficient numerical algorithm, except of course in the one-dimensional case. In other words, as soon as the 
type is multi-dimensional, $\theta=\left(\theta_{1}, \theta_{2}\right)$, we have no idea what the second-best security design looks like, and no efficient algorithm to compute the solution. The situation here is very different from the one described by Rochet and Choné [21], where they were able to give a complete solution in a (simple) two-dimensional case; to be sure, their mathematical problem was much simpler, and could readily be seen to have a unique solution, whereas in our case we do not have uniqueness, and proving existence was a mathematical challenge. The algorithms developed by Carlier, Lachand-Robert and Maury [8, 9] could probably be adapted to the present situation, but this is yet to be done.

More generally, we claim that most problems in adverse selection are inherently multidimensional, that is, the agent is fully described by the values of $k$ parameters $\theta_{1}, \ldots, \theta_{k}$, with $k \geq 2$. Most of the literature deal with the one-dimensional case, $k=1$, where the agent is characterized by the value of a single continuous parameter $\theta$, or even with the simpler case when $\theta$ can take only two values, $\underline{\theta}$ and $\bar{\theta}$. While this (excessive) simplification makes the mathematical model tractable, we would argue that it restricts the scope and power of the theory. In the case of an investor, for instance, it would not be reasonable to claim that he/she is fully characterized by his/her wealth and his/her risk aversion: the only defensible position is that he/she is characterized by both values together. In that sense, the two one-dimensional models we solved explicitly in the preceding section are academic: the only interesting one is the full two-dimensional model of Example 3.3. Unfortunately, it turns out to be a mathematical problem which has no precedent in the mathematical or physical literature, so the whole analysis has to be conducted from scratch, and we have been able to carry it through only to a certain point: we have shown that the solution exists, but we do not know what it looks like!

More precisely, let us note that we have allowed ourselves infinitely many instruments, while there are only finitely many types: indeed, the only restriction on the security $X$ to be designed is that it should belong to $L^{2}(\Omega)$, which is infinitely-dimensional unless $\Omega$ is finite. So any bunching which occurs will be purely endogenous, and cannot be attributed to the paucity of instruments. It would therefore be fascinating to see the rich interplay between the risk aversion and the risk exposure of the agents. Unfortunately, this is not possible at the present state of the art, for want of adequate mathematical tools. So it is quite urgent to develop efficient numerical methods for this new class of mathematical problems, in order to understand the economics of adverse selection in realistic cases.

The remaining ones stem from economics. The obvious question is: what about competition? We have assumed a monopoly situation, where the agent can deal only with one financial institution. This may be the case, to a certain extent, when the agent negotiates over the counter an option with his preferred bank, but then why does he not turn to another bank and get another quote? He may be restrained from doing so by informal transactions costs (shifting his account, running another set of negotiations, sharing con- 
fidential information with too many people). There may also be advantages for the agent in always dealing with the same bank: in an ongoing relationship, the bank learns about the agent, his past history and special needs, and this kind of information would be much more difficult to convey in a one-time transaction. In fact, there is empirical evidence to suggest that firms tend to stick with one financial intermediary: see for instance the paper $^{1}$ by Raghuan Rajan [18].

However, there is no denying that if we could have treated the competitive case, either from the market power or perfect competition perspective, we would have done it. Unfortunately, we do not know how to do so, thereby joining the vast majority of contributors to the literature on adverse selection, which is mainly confined to the monopoly situation. The main exception is, of course, the paper [23] by Rothschild and Stiglitz, which defines and studies equilibrium in a competitive insurance market with two types of agents (lowrisk and high-risk); it is hard to see how their results could be extended to a framework where there is a continuum of agents. Perhaps there will be some better idea in the future.

Finally, Pierre-André Chiappori has brought to our attention the fact that in the insurance industry, it is the principal, not the agent, who has private information. Large insurance companies who want to sell off part of their risk portfolio certainly know more about what they are selling than the prospective buyer. They have therefore to find a way to convince investors that they are not simply keeping good risks for themselves, and selling bad ones to an unsuspecting public. This problem seems extremely interesting to us, and we hope to investigate it in the near future. In summary, we hope that this paper does not close an avenue of research, but rather opens new ones.

\section{References}

[1] Akerlof G. (1970), The market for lemons, Quarterly Journal of Economics 84 (3) $488-500$

[2] Barrieu P. and ElKaroui N. (2005). Inf-convolution of risk measures and optimal risk transfer. Finance and Stochastics 9, 269-298.

[3] Bolton P., Dewatripont M. (2005) Contract theory, MIT Press

[4] Björk T. (2004) Arbitrage theory in continuous time, second edition, Oxford University Press

[5] Bühlmann, H. and S. Jewell (1979). Optimal risk exchanges. Astin Bulletin 10, 243262.

\footnotetext{
${ }^{1}$ We are indebted to the referee for pointing out this reference
} 
[6] Carlier G. (2001). A general existence result for the principal agent problem with adverse selection. Journal of Mathematical Economics 35, 129-150.

[7] Carlier G., Lachand-Robert T. (2001). Regularity of solutions for some variational problems subject to a convexity constraint, Comm. Pure Appl. Math., 54, 583-594.

[8] Carlier G., Lachand-Robert T. and Maury B. (2001). A numerical approach to variational problems subject to convexity constraints, Num. Math., 88, 299-318.

[9] Carlier G., Lachand-Robert T. and Maury B. (1999), $H^{1}$-projection into the set of convex functions: a saddle-point formulation, CEMRACS 1999 proceedings, ESAIM Proc. 10, 277-289.

[10] Duffie D. (2001) Dynamic asset pricing theory, third edition, Princeton University Press.

[11] Duffie D., Rahi R. (1995). Financial Market Innovation and Security Design: An Introduction, Journal of Economic Theory, 65 , 1-42.

[12] Gerber H.U. (1979). An Introduction to Mathematical Risk Theory. Huebner Foundation Monograph 8, Wharton School, University of Pennsylvania.

[13] Guesnerie R., Laffont J.-J. (1984). A complete solution to a class of principal agent problems with an application to the control of a self-managed firm, Journal of Public Economics, 25, 329-369.

[14] Jouini E., Schachermayer W. and Touzi N, Optimal risk sharing for law invariant monetary utility functions. Preprint.

[15] Kreps D. (1990) A course in microeconomic theory, Princeton University Press.

[16] Laffont J.J., Martimort D. (2001) The theory of incentives, Princeton University Press.

[17] Mussa M., Rosen S. (1978). Monopoly and Product Quality, Journal of Economic Theory, 18, 301-317.

[18] Rajan R. (1992). Insiders and Outsiders: The Choice between Informed and Arm'slength debt, Journal of Finance, 47, 1367-1400.

[19] Rochet J.-C. (1987). A necessary and Sufficient Condition for Rationalizability in a Quasi-linear context, Journal of Mathematical Economics, vol. 16.

[20] Rochet J.-C. (1989). Sur quelques problèmes de Calcul des Variations de l' Economie Mathématique, Annals of the CEREMADE, Birkhauser Ed.

[21] Rochet J.-C., Choné P.(1998). Ironing, Sweeping and Multidimensional screening, Econometrica, vol. 66 , 783-826. 
[22] Rockafellar R. T. (1970). Convex Analysis, Princeton University Press.

[23] Rothschild M., Stiglitz J. (1976) Equilibrium in competitive insurance markets, Quarterly Journal of Economics 90 (4) 629-649.

[24] Ruschendorf L. (2006). Risk measures for portfolio vectors and allocation of risks, preprint University of Freiburg.

[25] Salanié B. (1997) The economics of contracts: a primer, MIT Press.

[26] Spence M. (1973) Job market signalling, Quarterly Journal of Economics, 87 (3) 355-374. 